\title{
Compactación en suelos forestales: ¿mejor pezuñas o neumáticos?
}

\section{Forest soils compaction: better hoofs or rubber-tired?}

Aroca-Fernández, M.J. ${ }^{* 1}$; Bravo-Fernández, J.A. '; García-Viñas, J.I. ;

Fernández-Yuste, J.A. ${ }^{2}$; Serrada, R. ${ }^{3}$

1 Departamento de Sistemas y Recursos Naturales. Escuela Técnica Superior de Ingeniería de Montes Forestal $y$ del Medio Natural. Universidad Politécnica de Madrid. José Antonio Novais 10, 28040 Madrid.

2 Departamento de Ingeniería y Gestión Forestal Ambiental. Escuela Técnica Superior de Ingeniería de Montes Forestal y del Medio Natural. Universidad Politécnica de Madrid. José Antonio Novais 10, 28040 Madrid.

3 Sociedad Española de Ciencias Forestales, Pza. Pablo Iglesias 1, 19001 Guadalajara. 


\title{
Resumen
}

La compactación edáfica derivada de diferentes aprovechamientos forestales es actualmente tema de debate, particularmente en el contexto español en el que existe un nivel creciente de mecanización en la extracción de madera. Este trabajo tiene como objetivo comparar los niveles de compactación resultantes tras cinco décadas de tránsito de maquinaria durante los aprovechamientos mecanizados de madera (apeo con motosierra y saca con skidder de neumáticos), con el derivado del tránsito de ganado pastante (en el último siglo mayoritariamente vacuno en extensivo), en una masa de pino silvestre de la Sierra de Guadarrama sobre suelos franco-arenosos. Para ello se cuantifica la resistencia a penetración del suelo entre 0 y $50 \mathrm{~cm}$ de profundidad en 180 puntos repartidos aleatoriamente en el monte. Los resultados obtenidos muestran que: 1) no existen diferencias significativas en los primeros $50 \mathrm{~cm}$ entre los perfiles medios de resistencia a penetración del suelo generados por maquinaria y ganado en las zonas de alto tránsito; 2) dichas zonas sí muestran por el contrario diferencias significativas con las de bajo tránsito, que aumentan hasta los $16,8 \mathrm{~cm}$ de profundidad en que se alcanza el máximo (406,3 kPa); 3) a partir de esa profundidad la diferencia decrece hasta dejar de ser significativa en torno a $36,8 \mathrm{~cm}$. El caso aportado pone de manifiesto que, para predecir la compactación generada por un aprovechamiento en unas determinadas condiciones de estación, resulta imprescindible valorar no sólo el tipo de agente perturbador, sino también las características de las cargas y el régimen de perturbación previsto.

Palabras clave: aprovechamientos mecanizados, degradación edáfica, ganado, pino silvestre, resistencia a penetración.

\begin{abstract}
Soil compaction arising from forest resources exploitation is currently subject of recurrent debate, particularly in Spain, where there is an ongoing increase of logging mechanization level. The present study aims at analysing and comparing the soil compaction status resulting either from long-term machinery traffic during logging operations (ground based full tree logging with hand-felling, winching and skidder yarding) or from cattle traffic (extensively managed cows, mainly) within a scots pine forest located in the Spanish central mountains, over sandy loam soils, where both uses have been carried out for more than five decades. Soil penetration resistance was assessed from 0 to $50 \mathrm{~cm}$ depth in 180 points randomly selected within four 100x100 blocks throughout the forest. The results showed that: 1) after the long period of use, there were no significant differences between the average penetration resistance profiles $(0-50 \mathrm{~cm})$ of high traffic points of machinery and of cattle; 2) high and low traffic areas showed significant differences increasing till $16.8 \mathrm{~cm}$ depth where the maximum absolute disturbance was reached (406.3 $\mathrm{kPa}) ; 3$ ) from 16,8 $\mathrm{cm}$ depth on, differences decreased to become no longer significant at $36,8 \mathrm{~cm}$ depth. The present case shows that predictions concerning soil compaction effects of a forest use require taking into account not only the type of compacting agent, but also charges features and perturbations regimen.
\end{abstract}

Keywords: cattle, mechanized logging, penetration resistance, scots pine, soil degradation. 


\section{Introducción}

El suelo juega un papel clave en la relación del clima con la vegetación. Unas adecuadas condiciones edáficas de profundidad, textura, estructura y presencia de materia orgánica, optimizan la respuesta del ecosistema ante condiciones climáticas adversas, mejorando el comportamiento hídrico y térmico del mismo. La fragilidad del sistema ante los efectos negativos de fenómenos como la sequía y la torrencialidad, se ve de este modo atenuada o incrementada en función de las condiciones edáficas (Alaoui et al., 2018). Por este motivo, cuando la gestión forestal se plantea mejorar las condiciones de las masas para aumentar su resiliencia ante el endurecimiento de las condiciones climáticas, el suelo, y particularmente sus propiedades físicas, son un elemento clave a tener en cuenta. Es clave tener en cuenta su conservación y también sus posibilidades de mejora, pero para ello es imprescindible definir con rigor y base científica qué actuaciones y cuáles de sus elementos son los que le afectan y ponen en riesgo la sostenibilidad del sistema.

La compactación edáfica y los efectos que lleva asociados — disminución de la porosidad y empeoramiento de las condiciones de aireación, retención de agua y desarrollo radical (Miller et al., 2004)—, se citan frecuentemente en bibliografía como una de las causas de pérdida de calidad en los suelos que pueden afectar a la productividad de los mismos y en consecuencia al desarrollo vegetal (Kozlowski, 1999). Tanto el ganado como la extracción mecanizada de madera han sido tradicionalmente asociados a compactación edáfica en los suelos forestales. Si bien es cierto que en el contexto español de creciente nivel de mecanización en la extracción de la madera, es la maquinara el agente compactador más cuestionado, ambos forman parte de los aprovechamientos habituales en los montes españoles.

A través del análisis del estado de compactación de una masa forestal gestionada en extensivo durante más de 150 años para producción de madera y ganado (entre otros usos), el presente trabajo pretende comparar el impacto generado por ambos agentes compactadores y llevar a cabo una reflexión sobre los elementos a tener en cuenta a la hora de valorar el riesgo de compactación asociado a las actividades forestales.

\section{Material y métodos}

\subsection{Zona de estudio}

La zona experimental está localizada en el monte conocido como "Cabeza de Hierro", ubicado en la vertiente sur de la Sierra de Guadarrama ( $\left.40^{\circ} 50^{\prime} \mathrm{N}, 3^{\circ} 55^{\prime} \mathrm{W}\right)$, aproximadamente $45 \mathrm{Km}$ al norte de Madrid, entre 1260 y $2000 \mathrm{~m}$ de altitud. Presenta una precipitación media anual de $1115.8 \mathrm{~mm}$ (95.3 $\mathrm{mm}$ en la época estival) y una temperatura media anual de $7.7^{\circ} \mathrm{C}\left(24.1^{\circ} \mathrm{C}\right.$ de $\mathrm{T}^{\mathrm{a}}$ media de las máximas del mes más cálido y $-4.6^{\circ} \mathrm{C}$ de $\mathrm{T}^{\mathrm{a}}$ media de las mínimas del mes más frío). Los períodos de déficit hídrico y helada probable (Walter y Lieth, 1967) se alargan de junio a septiem- 
bre, y de noviembre a abril respectivamente. Los suelos son franco-arenosos (Roig Gómez, 1999) y están formados sobre roca madre de gneis (IGME, 1991).

El monte 'Cabeza de Hierro' tiene una extensión de 2016 ha (arboladas en un 94\%) y es propiedad de la Sociedad Anónima belga 'Pinares del Paular', que lo gestiona desde su adquisición en 1840. Está poblado por una masa de pino silvestre (Pinus sylvestris L.) irregular a escala de cantón, conformada por agregación de bosquetes homogéneos de edad y tamaño variables. En las zonas de menor altitud el estrato principal está salpicado de manchas de monte medio de rebollo (Quercus pyrenaica Willd.) dando lugar a una masa mixta. El sotobosque está compuesto por especies arbustivas entre las que destacan helechos (Pteridium aquilinum (L.) Kuhn), escobas (Genista sp.), enebros (Juniperus communis L.) y escaramujos (Rosa sp.). Debido al manejo y a las características de la estación, la masa presenta una elevada variabilidad a pequeña escala tanto en el estrato arbóreo como en el sotobosque (variación en densidad, edades, distribución espacial...) (Bravo-Fernández et al., 2009; Bravo-Fernández y Serrada Hierro, 2011).

El monte es gestionado de forma sostenible y rentable para objetivo múltiple: producción de madera, ganado, uso recreativo, protección de fauna y conservación ambiental. Entre estos usos, la producción de madera de pino silvestre ha sido uno de los objetivos principales de la gestión del monte desde 1840. Dicha gestión se ha realizado durante los últimos años por entresaca regularizada y aclareo sucesivo por bosquetes. Por su parte, el rebollo solía ser tratado mediante el método de beneficio de monte bajo para producción de leña, pero durante las últimas décadas se le viene aplicando un resalveo de conversión. Todos los tratamientos selvícolas han sido llevados a cabo de forma mecanizada durante los 50 años previos al muestreo del presente trabajo. Se ha empleado para ello el sistema de aprovechamiento de fuste completo con apeo manual y saca mediante skider con cabrestante. La maquinaria empleada en la mayoría de los casos ha sido un skidder Timberjack modelo 240A. La frecuencia aproximada de entrada de maquinaria para operaciones selvícolas es de una vez cada quince años. El monte está dotado con una red permanente de vías forestales así como arrastraderos, cargaderos y vías secundarias de saca.

El pastoreo en Cabeza de Hierro ha sido libre para el ganado local desde tiempos antiguos ya que, como señalan Bravo et al (2010), en el monte existía desde antes del Siglo XVII una servidumbre de pastos a favor de los vecinos del antiguo Sexmo de Lozoya de la Comunidad de Ciudad y Tierra de Segovia, sin limitación de especie, cargas, ni superficies pastables. Hasta los años sesenta, la cabaña ganadera pastante incluyó cabras, ovejas, caballos y vacas. Durante las últimas décadas ha pasado a estar integrada mayoritariamente por vacas (razas Negra avileña, Charolesa y Limusina) y en mucha menor proporción caballos. La carga pastante actual es aproximadamente de ciento cincuenta cabezas 8,5 meses al año (abril a noviembre). Los ungulados silvestres (corzos) también abundan en el monte.

\subsection{Diseño experimental y muestreo de datos}

El presente estudio ha sido llevado a cabo como parte de una investigación más 
amplia sobre la regeneración natural del pino silvestre en el entorno mediterráneo, para la que se contaba con un dispositivo experimental integrado por cuatro bloques de experimentación de 100x100 m ubicados en diferentes condiciones topográficas y de masa (tabla 1). En cada uno de esos bloques se seleccionaron aleatoriamente para este trabajo 48 puntos de muestreo en los que se realizaron mediciones de resistencia a penetración edáfica (RP) en pleno período de crecimiento vegetativo (mes de mayo) mediante un penetrómetro de cono RIMMIK CP20 $\left(30^{\circ}\right)$. Las inserciones de la sonda se llevaron a cabo, siempre que fue posible, hasta una profundidad de $49.5 \mathrm{~cm}$, registrando la resistencia a penetración cada $1.5 \mathrm{~cm}$. En los puntos de muestreo en los que el penetrómetro de cono interrumpió el registro antes de llegar a dicha profundidad, se repitió la medición hasta tres veces en $10 \mathrm{~cm}$ próximos al punto de inserción inicial. Cuando ninguna de las tres repeticiones alcanzó la profundidad objetivo, se tomó como definitiva la medición más profunda.

Tabla 1. Condiciones topográficas y de masa medias en los bloques experimentales.

\begin{tabular}{|c|c|c|c|c|c|}
\hline & & \multicolumn{4}{|c|}{ Bloque experimental } \\
\hline & & $\mathrm{A}$ & B & $\mathrm{C}$ & $\mathrm{D}$ \\
\hline Pendiente $(\%)$ & & 15.6 & 18.6 & 8.8 & 9.9 \\
\hline Altitud (m) & & 1526.7 & 1517.4 & 1441.4 & 1338.6 \\
\hline Orientación $\left(^{\circ}\right)$ & & 174.8 & 142.2 & 268.3 & 76.4 \\
\hline \multirow[t]{3}{*}{$\mathrm{N}\left(\mathrm{n}^{\mathrm{o}} \mathrm{pies} / \mathrm{ha}\right)$} & Psyl $_{10-20 \mathrm{~cm}}$ & 15,0 & 91,3 & 62,0 & 120,2 \\
\hline & Psyl $>20 \mathrm{~cm}_{1}$ & 173,6 & 169,0 & 156,5 & 257,2 \\
\hline & Qpyr & 202,8 & 498,5 & 47,9 & 60,8 \\
\hline \multirow[t]{3}{*}{$\mathrm{G}\left(\mathrm{m}^{2} / \mathrm{ha}\right)$} & Psyl $_{10-20 \mathrm{~cm}}$ & 0,3 & 1,5 & 1,2 & 2,3 \\
\hline & Psyl $_{>20 \mathrm{~cm}}$ & 38,8 & 26,3 & 21,5 & 26,5 \\
\hline & Qpyr & 3,9 & 11,4 & 2,2 & 1,5 \\
\hline \multirow[t]{3}{*}{$\mathrm{Dg}(\mathrm{cm})$} & Psyl $_{10-20 \mathrm{~cm}}$ & 14,9 & 14,3 & 15,2 & 15,2 \\
\hline & Psyl $_{20 \mathrm{~cm}}$ & 53,3 & 44,5 & 41,8 & 36,2 \\
\hline & Qpyr & 15,6 & 17,1 & 24,5 & 17,7 \\
\hline Altura dominante $(\mathrm{m})$ & Psyl & 24,7 & 22,4 & 18,7 & 26,4 \\
\hline \multirow[t]{2}{*}{ Altura media (m) } & Psyl & 20,0 & 15,6 & 13,9 & 16,4 \\
\hline & Qpyr & 10,2 & 10,7 & 11,9 & 10,2 \\
\hline
\end{tabular}

N: nºpies/ha; G: m² de área basimétrica normal/ha; Dg: diámetro medio cuadrático normal; Psyl10-20cm: pies de pino silvestre con $10 \mathrm{~cm}<$ $\emptyset_{1,3 \mathrm{~m}}<20 \mathrm{~cm}$; Psyl $>20 \mathrm{~cm}$; pies de pino silvestre con $\emptyset_{1,3 \mathrm{~m}}>20 \mathrm{~cm}$; Qpyr: todos los pies de rebollo con $\emptyset_{1,3 \mathrm{~m}}>10 \mathrm{~cm}$.

\subsection{Cuantificación del efecto de los agentes compactadores}

El nivel de tránsito de maquinaria y ganado en los distintos puntos de muestreo se estableció en función de su ubicación y el correspondiente historial de pastoreo y aprovechamientos mecanizados de la misma. Este tipo de análisis retrospectivo no permite establecer una clasificación excesivamente detallada de la intensidad de 
tráfico (Ezzati et al., 2012), ya que, si bien las áreas de alto impacto son fáciles de identificar, las de tránsito esporádico son mucho más difíciles de evaluar. Por este motivo, en el presente trabajo se diferenciaron únicamente dos categorías tanto para maquinaria como para ganado: "puntos de alta intensidad de tránsito" y "resto de puntos".

Se clasificaron como puntos de alta intensidad de tránsito de maquinaria todos los puntos de muestreo localizados sobre las vías de saca y los cargaderos presentes en los bloques experimentales. En general, la frecuencia media de uso de la red viaria viene siendo de una vez cada 2-3 años con un elevado número de pasadas en cada operación selvícola. Por el contrario, en el resto de superficie se entra aproximadamente una vez cada 15 años (cuando se realizan tratamientos en el rodal correspondiente) y las pasadas se reparten de forma aleatoria y mucho más reducida. Concretamente, según lo dispuesto en los planes de aprovechamientos del monte, en los rodales correspondientes a los bloques experimentales no se habían llevado a cabo tratamientos selvícolas mecanizados en los diez años anteriores al estudio; por el contrario, sí se había circulado por sus arrastraderos y vías de saca en los dos años anteriores al muestreo, en el transcurso de aprovechamientos en rodales adyacentes. Las características del tránsito de la maquinaria en las dos categorías consideradas se resumen en la tabla 2.

Tabla 2. Características del tránsito de maquinaria en las zonas de alto y bajo tránsito dentro de los bloques de experimentación.

\begin{tabular}{ccc}
\hline & \multicolumn{2}{c}{ Tránsito de maquinaria } \\
\cline { 2 - 3 } & Alto & Bajo \\
\hline Frecuencia de actuaciones mecanizadas & $2-3$ años & 15 años \\
Media de pasadas por actuación & Alta & Baja \\
Tiempo transcurrido desde la última actuación & $1-2$ años antes & $>10$ años antes \\
& del muestreo & del muestreo \\
\hline
\end{tabular}

Los niveles de tránsito de ganado se establecieron en función de la cobertura de herbáceas del suelo. Las zonas de elevada cobertura de herbáceas son las preferidas por el ganado para alimentarse, por lo que tienden a ser mucho más pisoteadas que aquellas recubiertas de matorral o restos vegetales (Sigua y Coleman, 2009; Tate et al., 2004). En la zona de estudio, a pesar de que existe pastoreo libre, el ganado tiende a concentrarse en torno a esas zonas de abundancia de pasto, especialmente en aquellas ubicadas junto a vías forestales o puntos de agua y/o suplementación (Bravo et al., 2010). Los cuatro bloques de experimentación están situados dentro del área de campeo del ganado y están salpicados por una proporción variable de pequeñas zonas de pastos en las que se postula que se concentra el pastoreo y el pisoteo de los animales. Por este motivo, para evaluar este pisoteo, se cuantificó el porcentaje de recubrimiento superficial de herbáceas en torno a cada punto de muestreo en parcelas de 1.5 x $1.5 \mathrm{~m}$, y se consideraron "puntos de alto tránsito" de ganado todos aque- 
llos que presentaron coberturas superiores al 90\%. Cabe destacar que en los bloques experimentales no existían zonas en las que por efecto de una excesiva carga ganadera el suelo hubiera perdido su recubrimiento vegetal, por lo que este tipo de superficies no suponían un problema para el método de clasificación planteado.

\subsection{Análisis de datos}

Como consecuencia de las características del perfil del suelo en la zona de estudio, la muestra de partida obtenida para el presente estudio incluyó puntos en los que no fue posible muestrear la RP hasta los $49.5 \mathrm{~cm}$. Sin embargo, se comprueba que el número de interrupciones en las mediciones no fue mayor en las capas más profundas y compactadas que en las superficiales. Tampoco se detectan valores de resistencia a penetración particularmente elevados en las profundidades previas a las paradas. Así, no se detecta una correlación significativa entre la frecuencia de interrupciones y la profundidad de muestreo, ni con el nivel de RP previo a la parada $(\rho<0.15$, p-values $>>0.05)$. La causa más probable de estas interrupciones es la presencia esporádica de grava, lo que ya ha sido mencionado en este tipo de estudios en el pasado (Miller et al., 2001; Salako, 2003), y encaja con la pedregosidad de pequeño tamaño observada en los perfiles de suelo. Se descarta por tanto el riesgo de sesgo en las mediciones debido a la eliminación sistemática de los tramos de muestra con valores extremos de RP, y se considera válida la muestra obtenida para representar el comportamiento edáfico en los 50 primeros centímetros de suelo. Aun así, para estudiar el comportamiento de la RP a lo largo del perfil de la forma más robusta posible a partir de la muestra disponible, se consideró adecuado analizar el comportamiento de la RP en profundidad a partir de perfiles completos, y no por tramos de profundidad tal y como se realiza en otros trabajos. Así, se optó por ajustar un modelo mixto para la RP incluyendo:

1) La profundidad como covariable.

2) Las perturbaciones (maquinaria y ganado) como factores fijos.

3) El punto de muestreo anidado en el bloque experimental como factor aleatorio.

La estructura óptima de efectos del modelo se seleccionó de acuerdo al procedimiento propuesto por Zuur et al. (2009).

En el caso de la estructura de efectos fijos se ajustaron y compararon cinco modelos (tabla 3) y, a través de estas comparaciones, se evaluó la significación del efecto de los distintos agentes compactadores. La valoración de la bondad de ajuste de los modelos se basó en el AIC (Akaike Information Criterion) y en el logaritmo de la verosimilitud (Log Likelihood).

El ajuste de los modelos se llevó a cabo mediante la función 'Ime' del paquete 'nlme' (Lindstrom y Bates, 1990, 1988) de R (versión 3.1.2.). 
Tabla 3. Lista de modelos a comparar para la optimización de la estructura de efectos fijos: Mi fórmula del modelo "i" (efectos fijos + efectos aleatorios); Gl: Grados de libertad.

\section{Efectos Fijos}

M0:

$\mathrm{Ti}$

M1:

M2:

$\mathrm{Ti}+(\mathrm{x}+\mathrm{x} 2+\mathrm{x} 3+\mathrm{x} 4)$

M3:

$$
\mathrm{Ti}+(\mathrm{x}+\mathrm{x} 2+\mathrm{x} 3+\mathrm{x} 4)+(\mathrm{AT})
$$

M4:

$$
\mathrm{Ti}+(\mathrm{x}+\mathrm{x} 2+\mathrm{x} 3+\mathrm{x} 4) *(\mathrm{AT})
$$

M5:
Efectos Aleatorios

Gl

$\begin{array}{lc}\text { x*Bloque/Punto } & 5 \\ \text { x*BloquePunto } & 9 \\ \text { x*BloquePunto } & 10 \\ \text { x*BloquePunto } & 14 \\ \text { x*BloquePunto } & 19 \\ \text { x*Bloque/Punto } & 24\end{array}$

$\mathbf{T i}=$ término independiente; $\mathbf{x}=$ profundidad de muestreo; $\mathbf{A} \mathbf{T}_{\mathrm{Maq}}=$ alto tránsito de maqunaria $(\mathrm{sí}=1, \mathrm{no}=0) ; \mathbf{A T}$ cat $=$ alto tránsito de ganado (sí=1, no=0); $\mathbf{A T}=$ alto tránsito de maquinaria o ganado (sí =1, no=0); Punto = punto de muestreo; símbolos para la descripción de la estructura del modelo: ' + ' = sólo se considera el efecto principal del factor indicado, '*' = se consideran tanto los efectos principales como las interacciones de los factores implicados), 'A/B' = efectos anidados (B en A).

\section{Resultados}

El ajuste de la estructura de efectos aleatorios del modelo reveló la existencia de un efecto significativo del punto de muestreo anidado en el bloque experimental, sobre la resistencia a penetración; dicho efecto resultó dependiente de la profundidad.

Por su parte, la comparación de modelos con diferente estructura de efectos fijos, una vez descontados los efectos aleatorios, puso de manifiesto que el modelo más adecuado (máxima sencillez sin pérdida significativa de capacidad explicativa) era el $\mathrm{M}_{3}$ (tabla 4). Dicho modelo sólo distingue entre puntos de alto impacto y de bajo impacto de perturbación $(\mathrm{AT}=1$ ó $\mathrm{AT}=0)$ y descarta la necesidad de

Tabla 4. Selección de la estructura del efectos fijos del modelo: $\mathbf{M}_{\mathrm{i}}$ : fórmula del modelo 'i' (efectos fijos + efectos aleatorios); Gl: grados de libertad; AIC: Akaike Information Critreria; logLik: 2*logaritmo(verosimilitud del modelo); L-ratio: estadístico para testar la mejora aportada por un modelo

\begin{tabular}{|c|c|c|c|c|c|c|c|}
\hline Efectos fijos & Efectos aleatorios & Gl & $A I C$ & $\log L i k$ & Test & L-ratio & p-value \\
\hline $\mathrm{M}_{0}: \mathrm{Ti}$ & $\mathrm{x}$ *Bloque/Punto & 5 & 71265.0 & -35627.5 & & & \\
\hline $\mathrm{M}_{1}: \mathrm{Ti}+\left(\mathrm{x}+\mathrm{x}^{2}+\mathrm{x}^{3}+\mathrm{x}^{4}\right)$ & $\mathrm{x}$ *Bloque/Punto & 9 & 70434.6 & -35208.30 & $\mathrm{M}_{\iota}$ vs $\mathrm{M}_{0}$ & 838.440 & $<0.001$ \\
\hline $\mathrm{M}_{2}: \mathrm{Ti}+\left(\mathrm{x}+\mathrm{x}^{2}+\mathrm{x}^{3}+\mathrm{x}^{4}\right)+(\mathrm{AT})$ & $\mathrm{x}$ *Bloque/Punto & 10 & 70385.5 & -35182.75 & $\mathrm{M}_{2} v s \mathrm{M}_{1}$ & 51.103 & $<0.001$ \\
\hline $\mathbf{M}_{3}: \mathbf{T i}+\left(\mathbf{x}+\mathbf{x}^{2}+\mathbf{x}^{3}+\mathbf{x}^{4}\right)^{*}(\mathbf{A T})$ & $\mathrm{x}$ *Bloque/Punto & 14 & 70380.4 & -35176.22 & $\mathbf{M}_{3} v s \mathbf{M}_{2}$ & 13.058 & 0.011 \\
\hline $\mathrm{M}_{4}: \mathrm{Ti}+\left(\mathrm{x}+\mathrm{x}^{2}+\mathrm{x}^{3}+\mathrm{x}^{4}\right)^{*}\left(\mathrm{AT}_{\mathrm{Maq}}+\mathrm{AT}_{\mathrm{Gan}}\right)$ & $\mathrm{x} *$ Bloque/Punto & 19 & 70380.2 & -35171.1 & $\mathrm{M}_{4} v s \mathrm{M}_{3}$ & 10.225 & 0.069 \\
\hline $\mathrm{M}_{5}: \mathrm{Ti}+\left(\mathrm{x}+\mathrm{xx}^{2}+\mathrm{x}^{3}+\mathrm{x}^{4}\right)^{*} \mathrm{AT}_{\mathrm{Maq}}+\mathrm{AT}_{\mathrm{Gan}}$ & $\mathrm{x}^{*}$ Bloque/Punto & 24 & 70388.1 & -35170.0 & $\mathrm{M}_{5} v s \mathrm{M}_{4}$ & 2.163 & 0.826 \\
\hline
\end{tabular}
con respecto al anterior más sencillo $\left(\mathrm{M}_{\mathrm{i}} v s \mathrm{M}_{\mathrm{i}-1}\right)$; $\mathbf{p}$-valor: significación asociada a dicho estadístico.

$\mathbf{T i}=$ término independiente; $\mathbf{x}=$ profundidad de muestreo; $\mathbf{A T}_{\mathrm{Maq}}=$ Alto Tránsito de maquinaria $(\mathrm{sí}=1, \mathrm{no}=0) ; \mathbf{A T}$ Gan $=$ Alto Tránsito de ganado $(s i ́=1$, no=0); AT $=$ Alto Tránsito de maquinaria o ganado $(s i ́=1$, no=0); Punto = punto de muestreo. Símbolos para la descripción de la estructura del modelo: ' $+{ }^{\prime}$ ' = sólo se considera el efecto principal del factor indicado, ${ }^{6 *}$ ' $=$ se consideran tanto los efectos principales como las interacciones de los factores implicados, 'A/B' = efectos anidados (B en A). cionado según el procedimiento propuesto por Zuur et al. (2009). 
tener en cuenta si la fuente de perturbación es maquinaria o ganado. El efecto del factor AT resultó significativo tanto sobre el término independiente, como sobre la pendiente de la relación entre la resistencia a penetración y la profundidad.

La expresión desarrollada del modelo óptimo $\mathrm{M}_{3}$ es la que se indica a continuación en la ecuación 1 :

Ecuación 1. Expresión desarrollada del modelo óptimo para describir en la zona de estudio el comportamiento de la resistencia a penetración en profundidad $(0-50 \mathrm{~cm})$ en función de la intensidad del tránsito de los agentes compactadores

$$
\begin{aligned}
\mathbf{R} \mathbf{P}_{i j} & =\beta_{0}+\left[\beta_{1} \boldsymbol{x}_{i j}+\beta_{2} \boldsymbol{x}^{2}{ }_{i j}+\beta_{3} \boldsymbol{x}^{3}{ }_{i j}+\beta_{4} \boldsymbol{x}^{4}{ }_{i j}\right]+\mathbf{A T} \cdot\left[\beta_{5} \boldsymbol{x}_{i j}+\beta_{6} \boldsymbol{x}^{2}{ }_{i j}\right. \\
& \left.+\beta_{7} \boldsymbol{x}^{3}{ }_{i j}+\beta_{8} \boldsymbol{x}^{4}{ }_{i j}\right]+\beta_{9} \mathbf{A} \mathbf{T}+\delta_{i}+\tau_{i} \boldsymbol{x}_{i j}+\boldsymbol{a}_{i j}+\gamma_{i j} \boldsymbol{x}_{i j}+\boldsymbol{e}_{i j}
\end{aligned}
$$

$\mathbf{R} \mathbf{P}_{i j}=$ resistencia a penetración en el punto de muestreo " $\mathrm{j}$ " del bloque experimental "i"; $\mathbf{x}_{i j}=$ profundidad de muestreo en el punto "j”" del bloque "i”; $\mathbf{A T}=$ Alto Tráfico de maquinaria o ganado $(\mathrm{s} i=1, \mathrm{no}=0) ; \boldsymbol{\beta}_{1 . . .9}$ $=$ coeficientes estimados para la covariable y los efectos fijos; $\boldsymbol{\delta}_{\mathrm{i}}=$ coeficiente estimado para el efecto aleatorio del bloque " $\mathrm{i}$ " sobre el término independiente del modelo; $\tau_{\mathrm{i}}=$ coeficiente estimado para el efecto aleatorio del bloque "i" sobre la pendiente del modelo. $\alpha_{\mathrm{ij}}=$ coeficiente estimado para el efecto aleatorio del punto " $\mathrm{j}$ " (perteneciente al bloque "i") sobre el término independiente; $\boldsymbol{\gamma}_{\mathbf{i}}=$ coeficiente estimado para el efecto aleatorio del punto " $\mathrm{j}$ ” (perteneciente al bloque " $\mathrm{i}$ ") sobre la pendiente.

Los coeficientes correspondientes a la parte fija del modelo (que es la que describe la relación entre la RP y la profundidad, una vez descontado el efecto aleatorio del bloque y el punto de muestreo), son los que se muestran en la tabla 5.

Tabla 5. Coeficientes de los efectos fijos del modelo ajustado para la estimación de la Resistencia a Penetración edáfica $(\mathrm{kPa})$ en función de la profundidad (modelo polinómico) teniendo en cuenta la intensidad del tránsito tanto de Ganado como de maquinaria.

\begin{tabular}{cccccc}
\hline & T. Independiente & $\mathrm{x}$ & $\mathrm{x}^{2}$ & $\mathrm{x}^{3}$ & $\mathrm{x}^{4}$ \\
\hline Alto tránsito & -82.51 & 208.67 & -8.87 & $20.07 \cdot 10^{-2}$ & $-17.34 \cdot 10^{-4}$ \\
Resto de puntos & 159.03 & 225.79 & -9.07 & $18.20 \cdot 10^{-2}$ & $-14.54 \cdot 10^{-4}$ \\
\hline
\end{tabular}

$\mathbf{x}=$ profundidad de muestreo

El modelo descrito equivale a dos ecuaciones para la RP, una para las zonas de alto tránsito $(\mathrm{AT}=1)$ y otra para el resto de puntos $(\mathrm{AT}=0)$. La representación gráfica de ambas curvas y de sus intervalos de predicción, junto con las series de valores medios de RP observados en función de la profundidad, se muestran en la figura 1.

De la estructura de efectos fijos obtenida para el modelo óptimo y de las curvas estimadas y sus correspondientes intervalos de predicción, se deduce que en los datos analizados se cumple que maquinaria y ganado generan un perfil de resistencia a pe- 


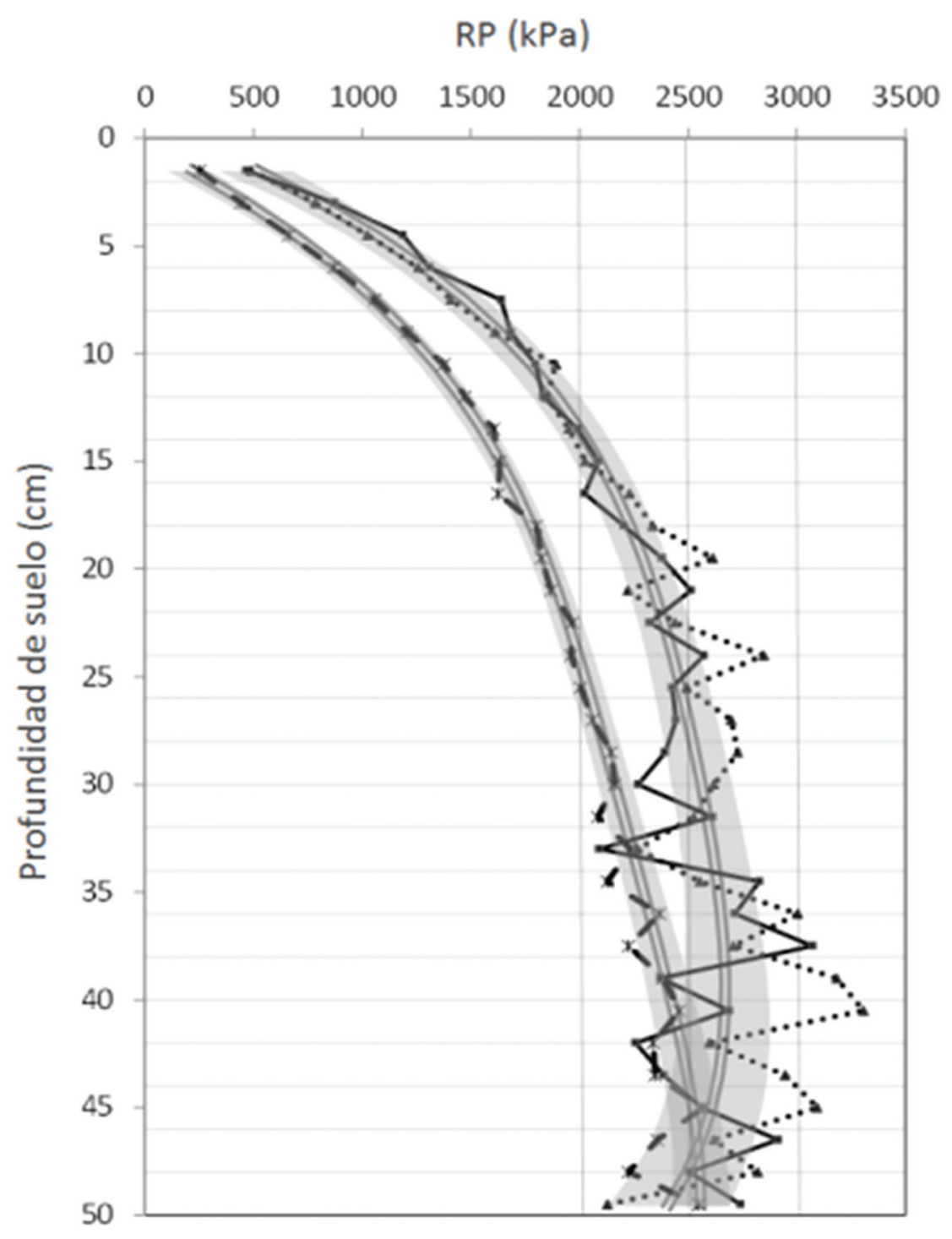

Figura 1. Mediciones medias vs estimaciones medias de RP en función de la profundidad y el tipo de tránsito: 1) Valores promedio de las mediciones de RP en función de la profundidad y el tipo de impacto: $-\mathbf{x}-$ Bajo tránsito, $\cdot \cdot \mathbf{\Delta} \cdot$ * Alto Tránsito de Maquinaria, $-\boldsymbol{-}-$ Alto Tránsito de Ganado. 2) Estimaciones del modelo mixto estimado: = Valores medios estimados de RP en función de la profundidad según el modelo mixto ajustado M3 (tablas 4-5). Intervalos de confianza para las predicciones medias del modelo.

netración en las zonas de alto tránsito significativamente diferente del de las zonas de bajo tránsito. Sin embargo, dentro de las zonas de alto tránsito no hay diferencias significativas entre los efectos medios de ambos agentes compactadores.

Así mismo, en la figura 1 puede observarse que las diferencias medias de RP entre puntos de alto y bajo tránsito van cambiando a lo largo del perfil. Si calculamos la función diferencia entre los modelos de 'Alto' y 'Bajo' tránsito (figura 2), 
encontramos que la variación de RP inducida por maquinaria o ganado va en aumento desde la superficie hasta $10 \mathrm{~s} 16.8 \mathrm{~cm}$, punto en el que se produce la perturbación máxima en valor absoluto $(406.3 \mathrm{kPa})$. A partir de los $16.8 \mathrm{~cm}$, la diferencia entre modelos disminuye hasta dejar de ser significativa, lo que, teniendo en cuenta los intervalos de predicción, se produce en torno a los $36.8 \mathrm{~cm}$ de profundidad.

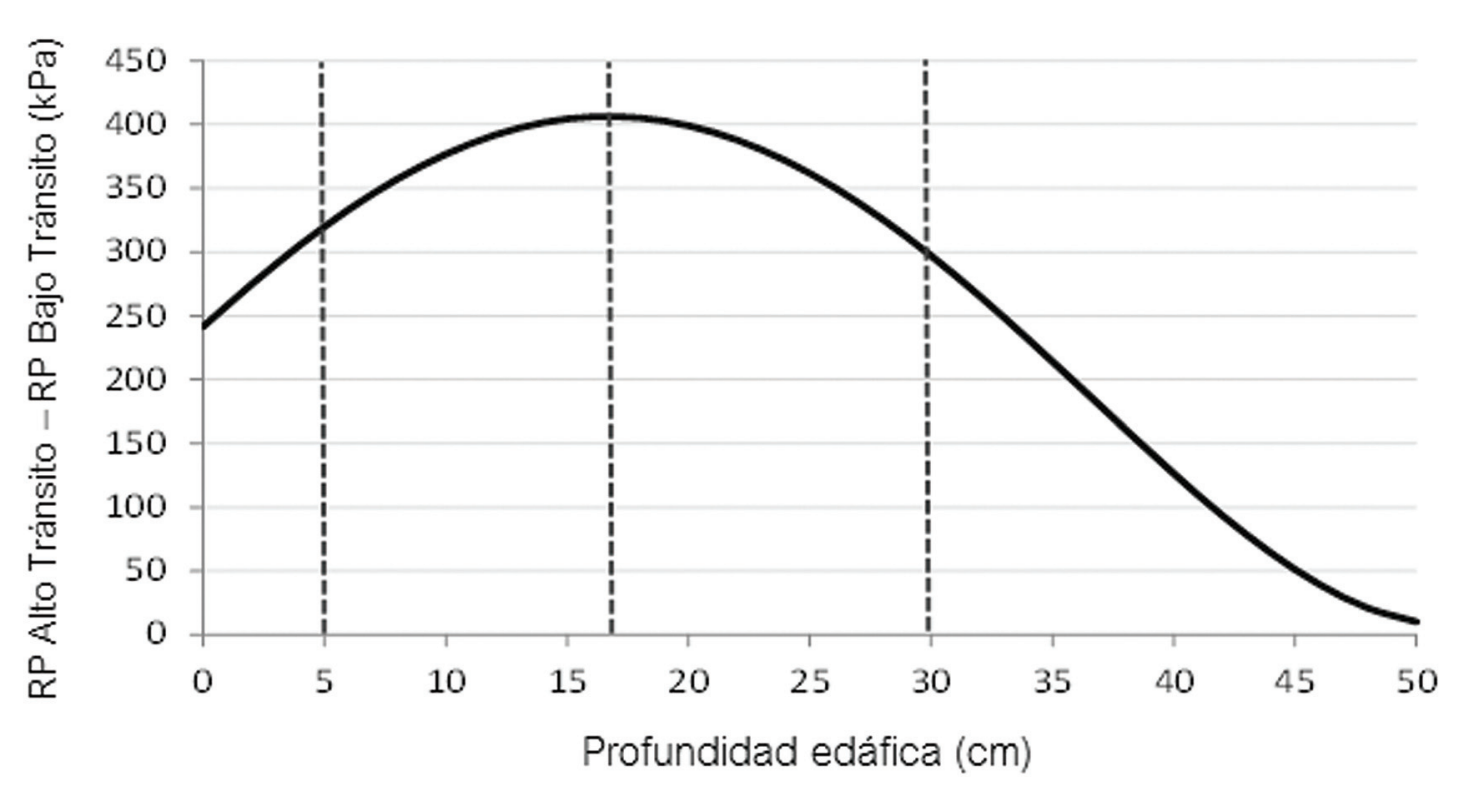

Figura 2. Curva diferencia entre el comportamiento medio estimado la resistencia a penetración (RP) para alto y bajo tránsito según el modelo mixto propuesto.

\section{Discusión}

Los niveles medios de resistencia a penetración observados a lo largo del perfil en la zona de estudio son intermedios con respecto a los rangos de valores encontrados en bibliografía para masas forestales de coníferas sobre suelos francos o franco arenosos (Ampoorter et al., 2007; Krzic et al., 2003; Mulholland y Fullen, 1991). Con respecto a los agentes compactadores, si bien es cierto que maquinaria y ganado presentan en algunos casos perfiles de compactación similares, es más habitual que se les atribuya diferente efecto sobre el suelo, particularmente en las capas más profundas (Sharrow, 2007). Así, tal y como puede observarse en la tabla 6 , en el caso de la maquinaria son más frecuentes niveles de compactación más elevados y más profundos que los encontrados en el área de estudio; y, por el contrario, el efecto del ganado (tabla 7) suele ser más superficial y en muchas ocasiones de menor intensidad, hasta el punto de que algunos autores afirman que no supera los 5-15 cm de profundidad (Greenwood y McKenzie, 2001). 


\begin{tabular}{|c|c|c|c|c|c|c|c|}
\hline 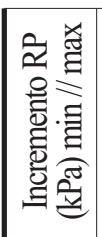 & $\stackrel{\circ}{0}$ & 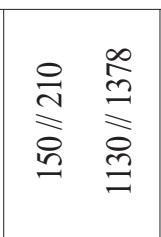 & 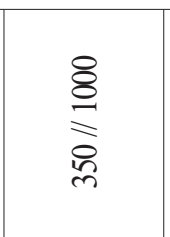 & 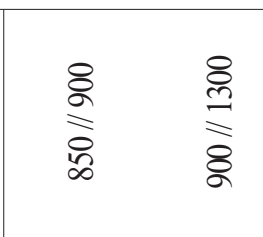 & 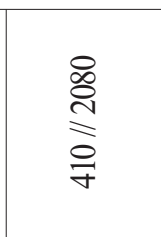 & $\underset{\substack{+\stackrel{8}{8}}}{\stackrel{8}{a}}$ & 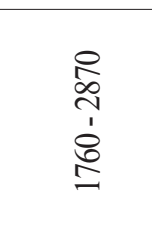 \\
\hline 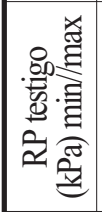 & $\stackrel{\infty}{n}$ & 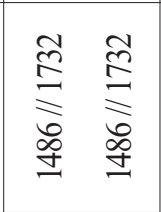 & $\stackrel{8}{\stackrel{8}{1}}$ & 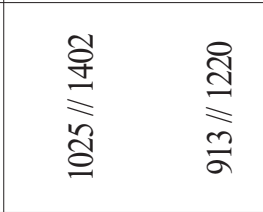 & 查 & 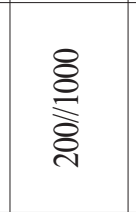 & 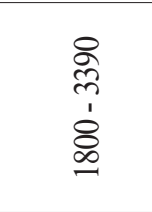 \\
\hline 岁急 & 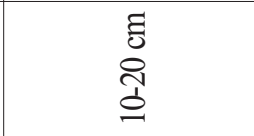 & $\begin{array}{cc}\tilde{E} & \Xi \\
0 & 0 \\
0 & 0 \\
\wedge 1 & \wedge\end{array}$ & 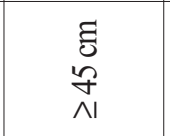 & $\begin{array}{l}\tilde{0} \\
0 \\
0 \\
\\
1\end{array}$ & $\begin{array}{l}\text { है } \\
\text { के }\end{array}$ & $\begin{array}{l}\tilde{0} \\
0 \\
0 \\
\Lambda\end{array}$ & 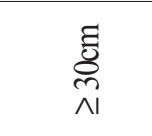 \\
\hline 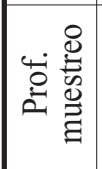 & $\begin{array}{l}\text { E् } \\
0 \\
+ \\
1 \\
0\end{array}$ & $\begin{array}{ll}\tilde{1} & \Xi \\
0 & 0 \\
0 & 0 \\
\tilde{0} & 1 \\
0 & 0\end{array}$ & $\begin{array}{l}\Xi \\
\vdots \\
\vdots \\
1 \\
0\end{array}$ & $\begin{array}{l}E \\
0 \\
0 \\
1 \\
0\end{array}$ & $\begin{array}{l}\tilde{0} \\
0 \\
\infty \\
1 \\
0\end{array}$ & 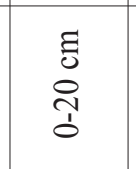 & $\begin{array}{l}\tilde{1} \\
0 \\
0 \\
0\end{array}$ \\
\hline 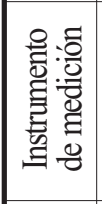 & 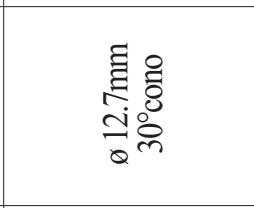 & 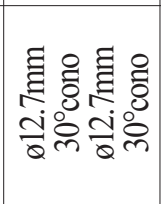 & 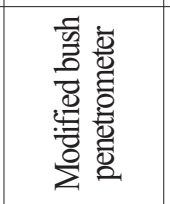 & 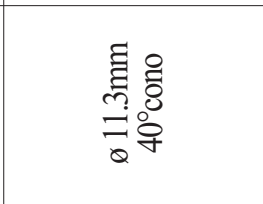 & 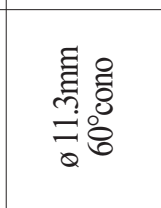 & $\begin{array}{l}\text { 圆 } \\
\text { 言 } \\
\text { †े } \\
\text { बे }\end{array}$ & 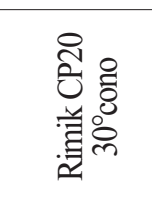 \\
\hline 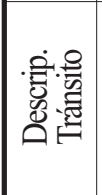 & 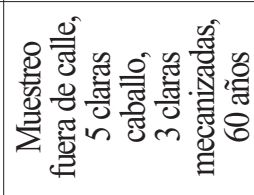 & 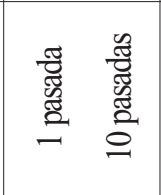 & 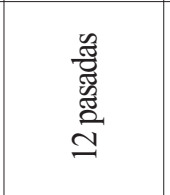 & 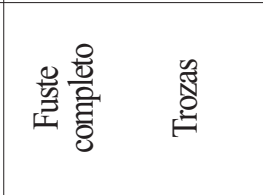 & 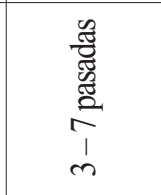 & 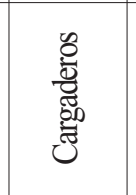 & 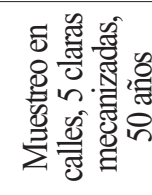 \\
\hline 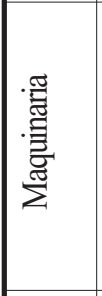 & 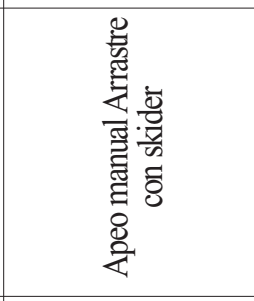 & 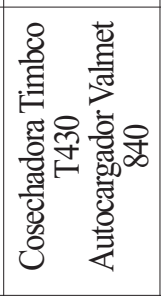 & 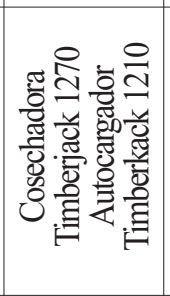 & 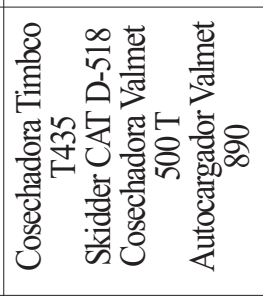 & 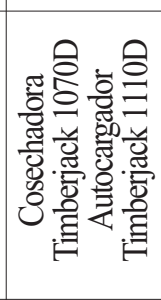 & & 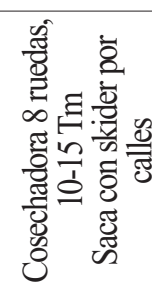 \\
\hline 畄哀 & 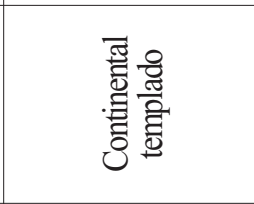 & 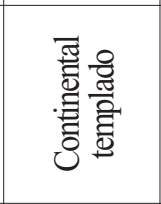 & 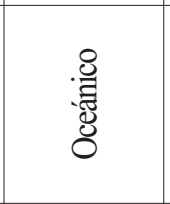 & 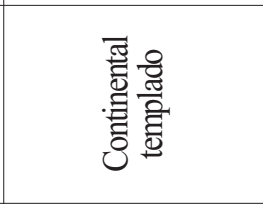 & 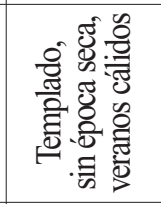 & 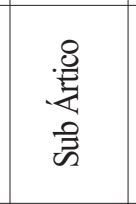 & 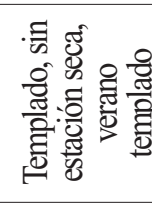 \\
\hline 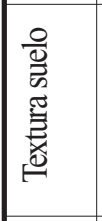 & 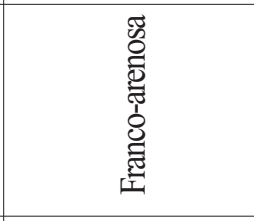 & 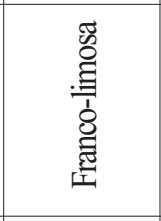 & 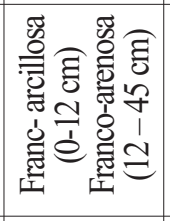 & $\begin{array}{l}\text { 马् } \\
\text { 蛋 }\end{array}$ & 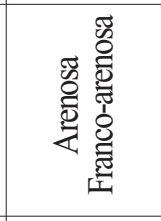 & 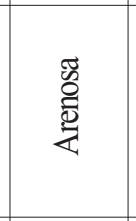 & 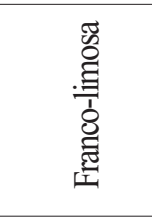 \\
\hline 亏్ّ & 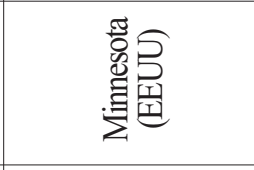 & 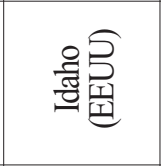 & 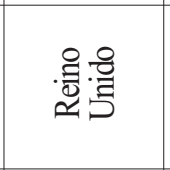 & 墕 & $\begin{array}{l}\text { 윰 } \\
\text { 흉 }\end{array}$ & 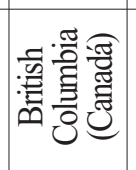 & $\begin{array}{l}\text { 㺃 } \\
\text { 胥 }\end{array}$ \\
\hline 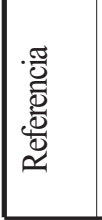 & 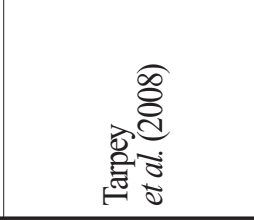 & 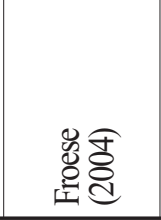 & 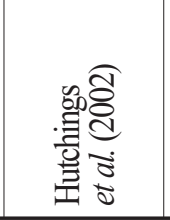 & 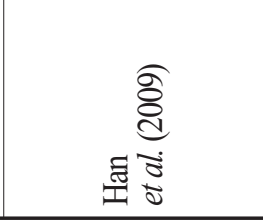 & 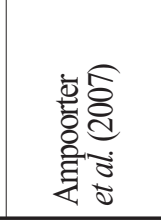 & 高这 & 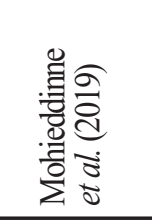 \\
\hline
\end{tabular}


Aroca-Fernández et al. | Cuad. Soc. Esp. Cienc. For. (2019) 45(3): 37-58

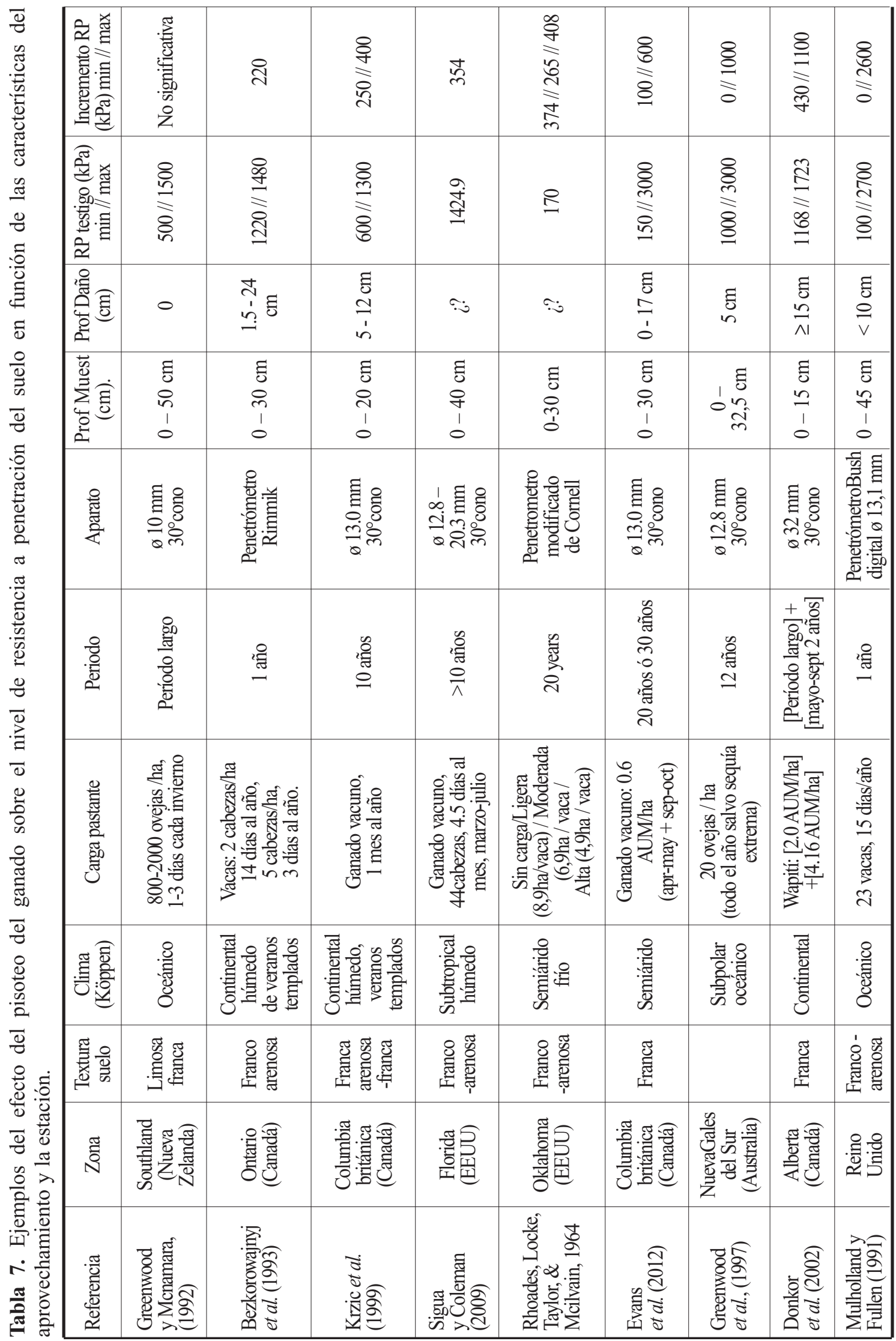


No obstante, tal y como se refleja en las tablas 6 y 7 , en la literatura se documentan una enorme variabilidad de efectos para cada uno de los dos agentes compactadores analizados. Dicha variabilidad pone de manifiesto que el agente compactador (maquinaria o ganado en nuestro caso) no define por sí sólo las características del impacto. Por el contrario, tal y como señalan Greacen y Sands (1980) tanto las características de la carga (peso, superficie de huella, tipo de presión), como las del régimen con el que actúa sobre el terreno (frecuencia de paso, total acumulado, patrón superficial de impacto, épocas de actuación...), son elementos clave para definir el impacto de una actividad sobre el estado de compactación del suelo bajo unas determinadas características de estación. Si bien es cierto que saber si la perturbación es ocasionada por ganado o por maquinaria acota en cierto modo estos aspectos, no los define completamente.

En lo referente a las características de la carga, frecuentemente se menciona en bibliografía que maquinaria y ganado ejercen presiones similares sobre el terreno (Greenwood y McKenzie, 2001), aunque la maquinaria tiene un efecto más intenso y más profundo debido a que ejerce la presión sobre una superficie mayor en cada pasada. Sin embargo, tanto maquinaria como ganado presentan mucha variabilidad en sus dimensiones. El tamaño de la hembra adulta de vaca de las razas de vacuno reconocidas y utilizadas en España, puede oscilar entre 180 y $925 \mathrm{~kg}$ en función de la raza y, si no restringimos por edad y sexo, la variación llega a ser entre 30 y 1350 kg (MAPA, 2019). En el caso de la maquinaria, los mismos tratamientos selvícolas pueden ser realizados (dependiendo del nivel de mecanización) por máquinas de entre 3000 y $30000 \mathrm{~kg}$ y, aun cuando nos limitamos a un solo tipo de máquina, encontramos que la presión estática sobre el suelo puede variar en más de un $75 \%$ tan sólo en función del tipo de neumáticos utilizado y de su presión de hinchado (Deschênes, 1989; Hakansson y Reeder, 1994; McDonald et al., 1995). Una comparativa bastante detallada de las características técnicas de carga sobre el terreno de una extensa lista de maquinaria forestal puede encontrarse en Deschênes (1989).

Con respecto al régimen de perturbación, hay que destacar la trascendencia que tiene en su repercusión final sobre el terreno el diferente patrón espacial de compactación de maquinaria y ganado. Así, mientras que la maquinaria compacta linealmente por bandas, el ganado compacta por puntos del tamaño de las pezuñas distribuidos de forma irregular dentro de la zona de pasto. Por este motivo, es corriente que la maquinaria genere un área de compactación en franjas razonablemente fáciles de identificar y con una intensidad de tránsito bastante homogénea. Sin embargo, la relación entre la carga pastante asignada a una zona de pasto y el número de pisadas sufridas por cada punto concreto, es mucho más variable (Drewry, 2006) y, salvo en casos de daño elevado, es muy complicado asignar con precisión espacial un gradiente de frecuencia de pisada. Así mismo, teniendo en cuenta la superficie total media pisada por una vaca al día y el número de pisadas necesarias para conseguir compactación máxima ( $\mathrm{n} \geq 100$ según Lei, 2004), todo parece indicar que, en el caso del ganado, hacen falta cargas pastantes o tiempos de permanencia elevados para que se alcancen sus niveles potenciales máximos de compactación.

Cuando el tiempo acumulado de aprovechamiento ganadero es lo suficiente- 
mente largo, el número de cabezas de ganado por unidad de superficie puede no influir en la intensidad de compactación alcanzada (Greenwood et al., 1997), puesto que la acumulación de efectos puede ser suficiente para alcanzar el equilibrio con la capacidad portante del suelo. No obstante, dado que en la mayoría de los estudios sobre compactación del ganado, los períodos contemplados son relativamente breves, es especialmente importante tener en cuenta el historial de perturbaciones (carga pastante + período total de permanencia del ganado), puesto que la probabilidad de encontrarnos por debajo del potencial máximo de perturbación de las cargas es elevada.

Si se analiza el efecto del ganado con cargas pastantes bajas y/o de corta duración, existe un alto riesgo de que la compactación observada corresponda a niveles bajos dentro del espectro de los posibles. Eso no implica que los sistemas no sean potencialmente más dañinos, sino que ese máximo potencial está aún lejos de materializarse. Sin embargo, a partir de los daños observados podría llegar a concluirse de forma imprecisa que el ganado pastante no puede generar daño más profundo o más intenso que el observado.

Por el contario, en el caso de la maquinaria, debido a su patrón de compactación por bandas y mayor índice de reiteración de paso, cabe esperar que se alcancen niveles máximos durante el transcurso de las operaciones forestales normales con mayor frecuencia, por lo que la compactación observada está mejor correlacionada con las características de las cargas y sus correspondientes potenciales de compactación.

En definitiva, la combinación carga-régimen de perturbación-estación nos sitúa en un escenario distinto cada vez y condiciona tanto el riesgo del sistema y la necesidad de intervención, como el tipo y probabilidad de éxito de las medidas a tomar. Por consiguiente, tanto las características de la carga como las del régimen de perturbación habrán de ser tenidas en cuenta a la hora a la hora de valorar y comparar la relevancia de los efectos de maquinaria y ganado.

En el caso del monte en estudio, las características de las cargas ejercidas por maquinaria y ganado en la zona de estudio se detallan en las tablas 8 y 9 .

Teniendo en cuenta los rangos de valores posibles mencionados anteriormente estaríamos ante ganado de tamaño medio alto, mientras que la maquinaria sería de tamaño medio bajo. Concretamente, El Timberjack 240E, que actualmente ya no se

Tabla 8. Características del ganado pastante en el área de estudio.

\begin{tabular}{cccc}
\hline Raza & $\begin{array}{c}\text { Peso hembra adulta } \\
(\mathrm{kg})^{*}\end{array}$ & $\begin{array}{c}\text { Estimación superficie pezuña } \\
\left(\mathrm{cm}^{2}\right) * *\end{array}$ & $\begin{array}{c}\text { Presión estática } \\
(\mathrm{kPa})\end{array}$ \\
\hline Negra Avileña & $500-600$ & 388,2 & 141,7 \\
Limousin & $650-850$ & 474,5 & 158,1 \\
Charolés & $700-950$ & 504,9 & 162,8 \\
\hline
\end{tabular}

*Fuente: Federación Española de Asociaciones de Ganado Selecto (FEAGAS) http://feagas.com/

** Área de pezuña estimada en base a un ajuste lineal sobre los datos peso-área de pezuña proporcionados por Greenwood and McKenzie (2001). 
Tabla 9. Especificaciones técnicas de la maquinaria empleada en el área de estudio (Deschênes, 1989).

\begin{tabular}{lcccc}
\hline Marca y modelo & $\begin{array}{c}\text { Peso }(\text { vacío }) \\
(\mathrm{kg})\end{array}$ & Neumáticos & \multicolumn{2}{c}{ Presión estática $(\mathrm{kPa})$} \\
\cline { 4 - 5 } & & & Sin carga & Con carga \\
\hline TimberJack Cable & 7441 & $23,1-26$ & & (Frontal) \\
Skidder 240A & & & 30 (Trasera) & \\
\hline
\end{tabular}

fabrica, tiene un peso muy inferior al de los nuevos modelos de arrastrador fabricados por John Deere.

En lo referente al historial de perturbación, en el caso del monte de estudio la carga pastante media teórica es baja $\left(0.075 \mathrm{UGM} \cdot \mathrm{ha}^{-1}\right)$. No obstante, la carga real sobre las zonas de concentración es mucho más elevada. Tal y como indican Bravo et al. (2010) en su estudio sobre el sobrepastoreo en el monte Cabeza de Hierro, a pesar de disponer de 2000 ha, el ganado concentra su actividad mayoritariamente en un $10-15 \%$ de la superficie del mismo. Este uso del territorio tan variable se explica por la estructura irregular por bosquetes de la masa, que genera a pequeña escala una mezcla de condiciones bastante distintas, de modo que se entremezclan en forma de mosaico: zonas abiertas, dominadas por pastos herbáceos, sin presencia de leñosas o con muy poca cobertura de éstas; y zonas dominadas por leñosas (arbolado o matorral), con el suelo poco iluminado y poca o nula presencia de pastos herbáceos con valor pastoral. Por otro lado, Moorefield and Hopkins (1951) señalan que habitualmente por término medio el ganado pasa prácticamente la mitad del tiempo en menos de un $10 \%$ de su área de pasto, por lo que cabe esperar que, dentro de ese $10-15 \%$ del monte, el pisoteo del ganado se reparta de nuevo de forma desigual concentrándose especialmente las zonas de mayor querencia. Si tenemos en cuenta estos porcentajes, la carga real esperada en las zonas de alto tránsito de ganado llegaría hasta las $3 \mathrm{UGM} \mathrm{ha}^{-1}$, lo que puede considerarse una carga elevada para explotaciones en extensivo (Ruiz et al., 2017). Así mismo, a partir de los tamaños de pezuña medios de la tabla 8 , y de la frecuencia media de 6300 pasos al día estimada para el ganado vacuno por Farris (1954), se puede aproximar de forma grosera que, por término medio, cada punto de la zona de alto tránsito ( $3 \mathrm{UGM} \cdot \mathrm{ha}^{-1}$ ) es pisado anualmente en torno a 10 veces. Ello implica que, a groso modo, hace falta una década para alcanzar en promedio las 100 pisadas que Lei (2004) estima necesarias para alcanzar el equilibrio de cargas con el suelo. Evidentemente, no es esperable el mismo efecto con 100 pisadas seguidas sobre un punto del terreno que con 100 pisadas espaciadas en 10 años, debido al diferente margen de actuación de los procesos de recuperación entre pisadas. Aun así, hay que tener en cuenta que el monte no ha sido pastado durante 10 años, sino durante más de 100 . No es descabellado por tanto pensar que el equilibrio carga-suelo puede haberse alcanzado para el ganado en la mayoría de los puntos de alto tránsito, y que el impacto que se observa en dichas zonas coincide ya con su potencial de compactación. Esto con- 
cuerda por otro lado con la elevada intensidad y profundidad observada para el efecto del ganado en el área de pasto con respecto al observado en otros lugares.

En el caso de la maquinaria, en cada rodal se han realizado por término medio tres intervenciones desde el inicio de los aprovechamientos mecanizados en el monte; a lo que hay que añadir en las zonas de alto tránsito analizadas — arrastraderos y cargaderos - todo el tránsito derivado de las operaciones en otros rodales a los que también dan servicio. Cabe esperar que en las zonas de alto tránsito también se hayan alcanzado sobradamente las 100 pasadas de la maquinaria. Sin embargo, en este caso, el estado de compactación observado está muy por debajo de los niveles máximos mencionados en bibliografía para los aprovechamientos mecanizados (tabla 6). Si bien es cierto que las condiciones de estación - suelo franco-arenoso profundo y bien estructurado, con presencia de abundante materia orgánica y un entramado radical adulto bastante denso y profundo- favorecen la resiliencia del sistema; también la forma de realizar los aprovechamientos ha permitido que el equilibrio suelo-carga se haya alcanzado en niveles mucho menores que en otros escenarios. Un aspecto importante en este sentido, es que dicho sistema de aprovechamiento con fuste completo, en el que el apeo y el desramado se realizan de forma manual y la saca se lleva a cabo mediante arrastre con un skider ligero, ha generado niveles de compactación mucho menores que los encontrados en otros aprovechamientos mecanizados, sin renunciar por ello a la sostenibilidad económica del aprovechamiento de la madera del monte.

\section{Conclusiones}

El caso aportado confirma el riesgo de compactación edáfica asociado al tránsito de maquinaria y ganado derivado de los aprovechamientos en los montes, al encontrar un aumento significativo del nivel de compactación en los primeros $35 \mathrm{~cm}$ de suelo de las zonas de mayor intensidad de paso. Sin embargo, al contrario que en una parte importante de la bibliografía, ambos agentes compactadores han generado en dichas zonas, similares perfiles medios de resistencia a penetración. Tanto las características de la maquinaria y el ganado empleado, como las del régimen con el que se han realizado ambos aprovechamientos, han sido claves en el impacto ocasionado.

En este sentido, cabe destacar la reducción de los niveles de compactación generados por la maquinaria con respecto a otros aprovechamientos mecanizados de madera en estaciones similares — a pesar del largo período de gestión acumulado-, gracias al empleo de maquinaria de peso medio-bajo para la saca, en combinación con apeo manual.

Por el contrario, se encuentra que no ha de menospreciarse el potencial compactador del ganado en extensivo cuando los aprovechamientos se prolongan durante períodos muy largos (más de 100 años en el monte analizado), aunque las cargas pastantes teóricas sean bajas. En este tipo de aprovechamiento ganadero, es fundamental tener en cuenta las diferencias entre cargas pastantes medias y puntuales, de- 
bido a la natural tendencia del ganado a concentrar el tránsito en torno a zonas de querencia y rutas fijas de desplazamiento entre ellas.

En cualquier caso, el ejemplo analizado pone de manifiesto que, a la hora de predecir la compactación generada por un aprovechamiento bajo unas determinadas condiciones de estación, no pueden extraerse conclusiones fiables partiendo únicamente del tipo de agente perturbador, sino que resulta imprescindible conocer las características de las cargas que supone y el régimen de perturbación previsto.

Señalar por último que todo lo expuesto anteriormente es válido para valorar el efecto de los agentes compactadores sobre los niveles de resistencia a penetración edáfica. No obstante, para analizar el efecto final de dichos niveles sobre la sostenibilidad de un monte, es necesario además valorar hasta qué punto afectan dichos cambios a la funcionalidad del mismo. Ello implica muchos más factores al margen de los señalados, empezando por la superficie total afectada en el conjunto del monte y su distribución espacial en el mismo.

\section{Bibliografía}

Alaoui, A.; Rogger, M.; Peth, S.; Blöschl, G.; 2018. Does soil compaction increase floods? A review. J. Hidrol. 557, 631-642. https://doi.org/10.1016/j.jhydrol.2017.12.052

Ampoorter, E.; Goris, R.; Cornelis, W.M.; Verheyen, K.; 2007. Impact of mechanized logging on compaction status of sandy forest soils. For. Ecol. Manage. 241, 162-174. https://doi.org/10.1016/j.foreco.2007.01.019

Bezkorowajnyj, P.G.; Gordon, A.M.; McBride, R.A.; 1993. The effect of cattle foot traffic on soil compaction in a silvo-pastoral system. Agrofor. Syst. 21, 1-10. https://doi.org/ 10.1007/BF00704922

Blouin, V.M.; Schmidt, M.G.; Bulmer, C.E.; Krzic, M.; 2005. Mechanical disturbance impacts on soil properties and lodgepole pine growth in British Columbia's central interior. Can. J. Soil Sci. 85, 681-691. https://doi.org/10.4141/S04-077

Bravo-Fernández, J.A.; Serrada Hierro, R.; 2011. «Cabeza de Hierro»: un monte privado, ordenado y ejemplo de multifuncionalidad. Foresta 52, 184-192.

Bravo-Fernández, J.A.; Serrada Hierro, R.; Aroca-Fernández, M.J.; 2009. Tercera Revisión de la Ordenación del monte «Cabeza de Hierro» (Rascafría, Madrid). En: $5^{\circ}$ Congreso Forestal Español, Ávila.

Bravo, J.A.; Rubio, A.; Aroca-Fernández, M.J.; Serrada, R.; Roig, S.; 2010. Diagnosis y distribución espacial del sobrepastoreo mediante Sig. Caso del monte "Cabeza de Hierro" (Rascafría, Madrid). En: IV Reunión Ibérica de pastos y forrajes. Zamora \& Miranda do Douro, pp. 537-542.

Deschênes, C.; 1989. Pression exerceé sur le sol par la machinerie forestiere: repertoire. Ministere de l'Energie et des Ressources, Canada, Quebec.

Donkor, N.; Gedir, J.; Hudson, R.; 2002. Impacts of grazing systems on soil compaction and pasture production in Alberta. Can. J. Soil Sci. 82, 1-8. https://doi.org/10.4141/ S01-008

Drewry, J.J.; 2006. Natural recovery of soil physical properties from treading damage of 
pastoral soils in New Zealand and Australia: A review. Agric. Ecosyst. Environ. 114, 159-169. https://doi.org/10.1016/j.agee.2005.11.028

Evans, C.R.; Krzic, M.; Broersma, K.; Thompson, D.J.; 2012. Long-term grazing effects on grasslands soil properties in southern British Columbia. Can. J. Soil Sci. 92, 685-693. https://doi.org/10.4141/cjss2011-070

Ezzati, S.; Najafi, A.; Rab, M.A.; Zenner, E.K.; 2012. Recovery of Soil Bulk Density, Porosity and Rutting From Ground Skidding Over a 20-Year Period after Timber Harvesting in Iran. Silva Fenn. 46, 521-538. https://doi.org/10.14214/sf.908

Farris, E.J.; 1954. Activity of dairy cows during estrus. J. Am. Vet. Med. Assoc. 125, 17120.

Froese, K.; 2004. Bulk density, soil strength, and soil disturbance impacts from a cut-tolength harvest operation in north central Idaho. Tesis doctoral. Univesity of Idaho.

Greacen, E.L.; Sands, R.; 1980. Compaction of forest soils. A review. Soil Res. 18, 163-189. https://doi.org/10.1071/SR9800163

Greenwood, K.L.; MacLeod, D. a.; Hutchinson, K.J.; 1997. Long-term stocking rate effects on soil physical properties. Aust. J. Exp. Agric. 37, 413-419. https://doi.org/10.1071/ EA96131

Greenwood, K.L.; McKenzie, B.M.; 2001. Grazing effects on soil physical properties and the consequences for pastures: a review. Aust. J. Exp. Agric. 41, 1231-1250. https://doi. org/10.1071/EA00102

Greenwood, P.R.; Mcnamara, R.M.; 1992. An analysis of the physical condition of two intensively grazed Southland soils. En: Proceedings of the New Zealand Grassland Association. pp. 71-75.

Hakansson, I.; Reeder, R.C.; 1994. Subsoil compaction by vehicles with high axle load extent, persistence and crop response. Soil Tillage Res. 29, 277-304. https://doi.org/10. 1016/0167-1987(94)90065-5

Han, S.-K.; Han, H.-S.; Page-Dumroese, D.S.; Johnson, L.R.; 2009. Soil compaction associated with cut-to-length and whole-tree harvesting of a coniferous forest. Can. J. For. Res. 39, 976-989. https://doi.org/10.1139/X09-027

Hutchings, T.R.; Moffat, A.J.; French, C.J.; 2002. Soil compaction under timber harvesting machinery: a preliminary report on the role of brash mats in its prevention. Soil Use Manag. 18, 34-38. https://doi.org/10.1079/SUM2002097

IGME, 1991. Mapa geológico de España, escala 1:50.000. Cercedilla. Instituto Tecnológico Geominero de España, Madrid.

Kozlowski, T.T.; 1999. Soil Compaction and Growth of Woody Plants. Scand. J. For. Res. 14, 596-619. https://doi.org/10.1080/02827589908540825

Krzic, M.; Newman, R.; Broersma, K.; 2003. Plant species diversity and soil quality in harvested and grazed boreal aspen stands of northeastern British Columbia. For. Ecol. Manage. 182, 315-325. https://doi.org/10.1016/S0378-1127(03)00064-1

Krzic, M.; Newman, R.; Broersma, K.; Bomke, A.; 1999. Soil compaction of forest plantations in interior British Columbia. J. range Manag. 52, 671-677. https://doi.org/10.23 $07 / 4003640$

Lei, S.A.; 2004. Soil compaction from human trampling, biking, and off-road motor vehicle activity in a Blackbrush (Coleogyne ramossisima) shrubland. Weswtern North Am. Nat. 64, 125-130.

Lindstrom, M.J.; Bates, D.M.; 1988. Newton-Raphson and EM Algorithms for Linear Mo- 
dels for Repeated-Measures Data. J. Am. Stat. Assoc. 83, 1014-1022. https://doi.org/ 10.1080/01621459.1988.10478693

Lindstrom, M.J.; Bates, D.M.; 1990. Nonlinear mixed effects models for repeated measures data. Biometrics 46, 673-687. https://doi.org/10.2307/2532087

MAPA, 2019. Catalogo Oficial de Razas de Ganado de España [WWW Document]. URL https://www.mapa.gob.es/es/ganaderia/temas/zootecnia/razas-ganaderas/razas/catalo go/ Accedido 13 septiembre 2019.

McDonald, T.P.; Stokes, B.J.; Aust, W.M.; 1995. Soil physical property changes after skidder traffic with varying tire widths. J. For. Eng. 6, 41-50. https://doi.org/10.1080/084352 43.1995.10702671

Miller, R.E.; Colbert, S.R.; Morris, L.A.; 2004. Effects of heavy equipment on physical properties of soils and on long-term productivity: a review of literature and current research. Nactional Council for Air and Stream Improvement.

Miller, R.E.; Hazard, J.; Howes, S.; 2001. Precision, Accuracy, and Efficiency of Four Tools for Measuring Soil Bulk Density or Strength. U.S.Department of Agriculture Forest Service, Portland, OR. https://doi.org/10.2737/PNW-RP-532

Mohieddinne, H.; Brasseur, B.; Spicher, F.; Gallet-moron, E.; Buridant, J.; Kobaissi, A.; Horen, H.; 2019. Physical recovery of forest soil after compaction by heavy machines, revealed by penetration resistance over multiple decades. For. Ecol. Manage. 449, 1-10. https://doi.org/10.1016/j.foreco.2019.117472

Moorefield, J.; Hopkins, H.; 1951. Grazing habits of cattle in a mixed-prairie pasture. $J$. Range Manag. 4, 151-157. https://doi.org/10.2307/3893696

Mulholland, B.; Fullen, M. a.; 1991. Cattle trampling and soil compaction on loamy sands. Soil Use Manag. 7, 189-193. https://doi.org/10.1111/j.1475-2743.1991.tb00873.x

Rhoades, E.D.; Locke, L.F.; Taylor, H.M.; Mcilvain, E.H.; 1964. Water intake on sandy range as affected by 20 years of differential cattle stocking rates. J. Range Mgt 17, 185 190. https://doi.org/10.2307/3895762

Roig Gómez, S.; 1999. Caracterización edáfica de los principales pastizales naturales del valle del Paular (Madrid). Tesis doctoral. Universidad Politécnica de Madrid.

Ruiz, J.; Herrera, P.M.; Barba, R.; Busqué, J.; 2017. Definición y caracterización de la extensividad en las explotaciones ganaderas en España. Ministerio de Agricultura y Pesca, Alimentación y Medio Ambiente. 98 pp.

Salako, F.K.; 2003. Soil physical conditions in Nigerian Savannas and biomass production. Tesis doctoral. University of Agriculture, Abeokuta, Nigeria.

Sharrow, S.H.; 2007. Soil compaction by grazing livestock in silvopastures as evidenced by changes in soil physical properties. Agrofor. Syst. 71, 215-223. https://doi.org/10.1007/ s10457-007-9083-4

Sigua, G.C.; Coleman, S.W.; 2009. Long-term effect of cow congregation zone on soil penetrometer resistance: implications for soils and forage quality. Agron. Sustain. Dev. 29, 517-523. https://doi.org/10.1051/agro/2009021

Tarpey, R.A.; Jurgensen, M.F.; Palik, B.J.; Kolka, R.K.; 2008. The long-term effects of silvicultural thinning and partial cutting on soil compaction in red pine (Pinus resinosa Ait.) and northern hardwood stands in the northern Great Lakes Region of the United States. Can. J. Soil Sci. 88, 849-857. https://doi.org/10.4141/CJSS08001

Tate, K.W.; Dudley, D.M.; Mcdougald, N.K.; Melvin, R.G.; 2004. Effect of canopy and grazing on soil bulk density. Rangel. Ecol. Manag. 57, 411-417. https://doi.org/10.2111/ 
1551-5028(2004)057[0411:EOCAGO]2.0.CO;2

Walter, H.; Lieth, H.; 1967. Klimadiagramm-Weltatlas, Jena, VEB. ed.

Zuur, A.; Leno, E.N.; Walker, N.; Saveliev, A.A.; Smith, G.M.; 2009. Mixed effects models and extensions in ecology with $R$. Springer-Verlag New York, New York. https://doi. org/10.1007/978-0-387-87458-6 
